Sánchez Torrejón, B. (2021). La formación del profesorado de Educación Primaria en diversidad sexo-genérica. Revista Electrónica Interuniversitaria de Formación del Profesorado, 24(1), 253-266.

DOI: https://doi.org/10.6018/reifop.393781

\title{
La formación del profesorado de Educación Primaria en diversidad sexo-genérica
}

\author{
Begoña Sánchez Torrejón \\ Facultad de Ciencias de la Educación. Universidad de Cádiz (España)
}

\section{Resumen}

El presente trabajo de investigación surge del objetivo de detectar la formación del profesorado en activo de la etapa de Educación Primaria sobre la diversidad sexo-genérica y su intervención con respecto a la inclusión del alumnado no heteronormativo en los centros escolares. Es un elemento clave en la inclusión del alumnado no heteronormativo la formación del profesorado en diversidades sexo-genéricas para la construcción de una escuela libres de LGTBfobias. Hemos utilizado la metodología cualitativa, concretamente el instrumento planteado para recabar la información ha sido la entrevista. La muestra del universo poblacional está compuesta por 40 profesores y profesoras en activo de Educación Primaria de la provincia de Cádiz de colegios públicos. Entre los resultados más destacados se refleja la confusión sobre la temática unido a la escasa formación, por lo cual el profesorado no tiene herramientas para incluir la diversidad sexo-genérica en los centros escolares. Se detecta que todavía es un tema tabú en la escuela y que crea situaciones de rechazo al plantearse. Destacamos el interés por parte del profesorado en recibir formación sobre la temática para poder trabajar de manera inclusiva la diversidad sexo-genérica.

\section{Palabras clave}

Diversidad sexo-genérica; educación primaria; formación del profesorado; inclusión.

\section{Contacto:}

Begoña Sánchez Torrejón, begonia.sanchez@uca.es, Facultad de Ciencias de la Educación. Universidad de Cádiz. Avenida Saharaui s/n. Campus Universitario Río San Pedro. C.P. 11519 Puerto Real. Cádiz. 


\title{
The training of Primary Education teachers in gender diversity
}

\begin{abstract}
This research work arises from the goal of detecting the training of active teachers in Primary Education on gender diversity and its intervention with respect to the inclusion of non-heteronormative students in schools. A key element in the inclusion of nonheteronormative students is the training of teachers in gender diversity for the construction of a school free of LGTBphobia. We have used the qualitative methodology, specifically the instrument proposed to collect the information has been the interview. The sample of the population universe is made up of 40 active Primary Education teachers from the province of Cadiz from public schools. Among the most notable results is reflected the confusion on the subject along with poor training, so teachers have no tools to include gender diversity in schools. We found that it is still a taboo subject at school and that it creates situations of rejection when it arises. We highlight the interest expressed by the teachers in receiving training on the subject in order to be able to work with gender diversity in an inclusive manner.
\end{abstract}

\section{Key words}

Gender diversity; primary education; teacher training; inclusion.

\section{Introducción}

Los centros educativos, como espacios de socialización, tienen un papel clave en la socialización de la orientación sexual y la identidad de género del alumnado, sobre todo en edades tempranas como las que caracterizan a la etapa de Educación Primaria; sin embargo, no suelen ser conscientes de que sus modelos de interacción puedan ser discriminatorios y estos carecen de las estrategias necesarias para cambiar sus conductas sesgadas desde el modelo heteronormativo:

La escuela, al ser la institución de reproducción de la cultura por excelencia tiene un papel importante en la construcción de valores de tolerancia y respeto, pero también precisamente por su papel socializador, tiende a reproducir estereotipos y estigmas frente a aquellos considerados diferentes, como es el caso de las y los jóvenes con preferencia sexual distinta a la heterosexual (Lara y Ochoa, 2007, p.10).

Estamos ante un sistema educativo heterosexista, ya que solo contempla como modelo referente la heterosexualidad. Con ello oculta intencionadamente las diversidades sexogenéricas, invisibilizándolas y excluyendo a todo el alumnado que no desempeña los roles dominantes ajustados a la heteronormatividad. La educación en la diversidad sexo-genérica está ausente de la mayoría de los centros de enseñanza en los que, lejos de educar al alumnado en esta diversidad, se tiende a favorecer una visión heterosexista y homófoba de la sexualidad. Visión homófoba que es una consecuencia inevitable del tabú social y educativo existente en torno a todos estos temas (Borrillo, 2001).

La diversidad sexo-genérica es un tipo de diversidad más al que hay que dar una respuesta educativa de calidad desde los centros de enseñanza. El desarrollo afectivo-sexual del alumnado es una parte más de su desarrollo integral, cuestión clave que no podemos olvidar en nuestros centros educativos. La heteronormatividad que se trasmiten en los 
centros escolares impuestas por el modelo hegemónico comienzan a hacerse presentes desde temprana edad, repercuten directamente sobre la forma en la que las niñas y los niños se autoperciben y cómo ven a los demás, perpetuando la desigualdad y las discriminaciones de género desde la infancia.

Por su parte, Goldfarb (2006) analizó la homofobia verbal en la etapa de Educación Primaria y encontró que en esta etapa el empleo de insultos homófobos era muy frecuente. Con respecto a las causas de este alto índice de empleo de insultos homófobos en el alumnado, Goldfarb (2006) lo achacaba al alto empleo que existe a nivel social de los mencionados insultos, es decir, el aula no deja de ser, para esta autora, un reflejo de lo que ocurre fuera del aula, a nivel social.

Plumier (2001) plantea que los insultos que se den desde la Educación Primaria, antes de que el alumnado haya desarrollado su orientación sexual, tienen graves consecuencias, ya que estos condicionan la construcción de la identidad sexual no solo de la persona agredida, sino también de quienes oyen aquellos insultos.

\section{La formación del profesorado en diversidad sexo-genérica}

Un aspecto clave en el buen funcionamiento y mejora de los centros escolares es la formación del profesorado, como se recoge en el artículo 102 de la LOMCE. El profesorado debe ir acompañado de un conjunto de procesos formativos que le ayude a reciclarse profesionalmente, así como por una serie de recursos que les permitan poder desarrollar correctamente su labor y enfrentarse a las diferentes situaciones que puedan llegar a condicionar su práctica docente.

Puede decirse que la formación permanente constituye un derecho y una obligación de todo el profesorado y una responsabilidad de las Administraciones educativas y de los propios centros. Para lograrlo se debe contar con una formación del profesorado que incorpore en todas sus etapas, inicial y continua, contenidos que promuevan la igualdad como elementos básicos para la lucha contra la discriminación y estereotipos sexuales, contenidos actualizados para proporcionar herramientas para la inclusión del diversidad afectivo-sexual en el aula y en el centro educativo.

Se pone así de relieve la necesidad de que el alumnado exprese su orientación sexual e identidad de género de manera libre en las aulas, y que la comunidad educativa propicie un ambiente respetuoso y sea facilitadora del desarrollo afectivo-sexual de todo el alumnado:

El aula puede transformarse en un espacio que favorezca el cambio social si la práctica docente conjuga una revisión de la estructura autoritaria que suele definir sus estrategias $y$, sobre todo, con el cuestionamiento cotidiano de la heterosexualidad normativa a través del modelo de aprendizaje trasgresor (Britzman, 2002, p. 25).

Elsbree (2002) destaca la importancia de la formación del profesorado para la atención a la diversidad sexo-genérica como factor de inclusión. El profesorado es el protagonista en el papel de propiciar contextos educativos en los que se desarrollan las competencias a través de metodologías que posibiliten el diálogo, las interacciones, la solidaridad, la igualdad y el respeto a todo el alumnado, y que propicie una experiencia inclusiva donde cada persona sea reconocida y valorada, donde cada una se sienta apoyada, independientemente de cuál sea su orientación sexual e identidad de género.

Muchos son los autores y las autoras que denuncian la falta de formación del profesorado en esta temática. López Soler (2003) apunta que en los planes de formación permanente del profesorado de Educación Infantil y Educación Primaria no existen materias específicas para tratar cuestiones relacionadas con la educación sexual. Desde la formación inicial, los 
docentes carecen de una formación adecuada respecto a la intervención de la diversidad sexo-genérica en el contexto escolar (Gallardo y Escolano, 2009).

Como resalta Sofía Díaz de Greñu (2010), uno de los grandes obstáculos que encontramos en la actualidad para normalizar el tratamiento de la cuestión de la diversidad de género en los centros educativos son los numerosos estereotipos negativos que tienen el profesorado y la insuficiente formación recibida por parte de este sobre esta temática.

Wynee (2008) relaciona la falta de educación en la diversidad sexo-genérica en los centros educativos, con la falta de formación del profesorado para la atención a este tipo de diversidad. Esta falta de formación que tiene su correlato más evidente en la falta de propuestas formativas de esta temática por parte de las administraciones educativas. Vemos consecuentemente reflejado cómo la formación del profesorado con relación a la diversidad sexo-genérica en la actualidad es escasa y generalmente, inexistente:

La falta de formación específica se convierte en un hándicap que se hace más evidente cuando hay acoso como consecuencia de cuestiones de género o sexualidad, situaciones ante las cuales una parte nada desdeñable del profesorado dice no actuar porque no sabe cómo hacerlo o no se siente con la seguridad suficiente para intervenir (Pichardo, 2015, p. 118).

A tenor de lo expuesto, insistimos en que la formación del profesorado es un elemento clave para la mejora de la calidad educativa y con respecto a la temática de esta investigación, la diversidad sexo-genérica, es necesaria para que el profesorado de Educación Primaria este formado en este ámbito y pueda propiciar una escuela inclusiva. La participación activa del profesorado es imprescindible para construir entre todas y todos una educación inclusiva, sensible con la diversidades sexo-genéricas y a las necesidades del alumnado, respetando la pluralidad y comprometida con la inclusión de todo el alumnado en los centros escolares.

\section{Metodología}

A continuación, nos centraremos en la metodología de investigación, que ha sido la investigación cualitativa. Esta modalidad nos aproxima a las interacciones humanas, a la comprensión de los procesos sociales y de las subjetividades e intersubjetividades de quienes constituyen parte del proceso investigativo. Hemos elegido esta metodología por que valoramos la necesidad de dialogar y escuchar activamente al profesorado con respecto a la diversidad sexo-genérica, pues consideramos que es un tema que necesita visibilizarse con todas las posibles visiones y aportaciones por el propio profesorado en activo.

La investigación cualitativa da protagonismo a las personas participantes o informantes, interactuando directamente con ellas: "el investigador tiene las preguntas y el sujeto de la investigación tiene las respuestas" (Taylor y Bogdan, 1984, p. 101); no recurre a pruebas estandarizadas que se caracterizan precisamente por el anonimato y el manejo de grandes muestras de población.

Toda investigación parte de unos objetivos que representan lo que se quiere hacer, lograr, o, simplemente, analizar. El objetivo general de esta investigación de acuerdo con la problemática objeto de estudio es conocer la formación del profesorado de la etapa de Educación Primaria sobre las diversidades sexo-genéricas. Este objetivo general de la investigación se concreta en varios objetivos específicos: Conocer las ideas que poseen sobre las sexualidades no normativas. Identificar las carencias formativas iniciales que tiene 
la comunidad educativa de Educación Primaria sobre la diversidad sexo-genérica. Descubrir las intervenciones educativas que se llevan a cabo sobre la diversidad sexo-genérica en los centros educativos en la Educación Primaria. Analizar el modelo sexual que se transmite en Educación Primaria.

\section{Participantes}

La muestra entrevistada ha sido elegida de manera aleatoria del universo poblacional que forma el total de centros escolares públicos de la provincia de Cádiz de Educación Primaria, se ha entrevistado al profesorado de sexto de primaria. Para la investigación que se han entrevistado a 40 profesores y profesoras, 11 profesores y 29 profesoras de edades comprendidas entre 28 y 51 años.

El ejercicio de la investigación científica y el uso del conocimiento producido por la ciencia demandan conductas éticas. En las principales cuestiones éticas relacionadas con el proceso de investigación cualitativa nos exige considerar tres principios éticos: el consentimiento informado (Powell y Single, 1996), con el que donde las personas participantes de la investigación permiten participar y compartir su información. Otra de las cuestiones éticas es la privacidad y la confidencialidad de la información, cuestiones que son primordiales para garantizar la intimidad de las personas. Todos los participantes deben dar su aprobación para ser grabados, a la par dentro del consentimiento se debe de garantizar la total confidencialidad, junto con el anonimato y la seguridad, tanto actual como futura, de la información que se trasmita.

\section{Instrumento}

La entrevista persigue el propósito, de obtener una determinada información (Vázquez y Angulo, 2003). Pero, en cualquier caso, el objetivo último es acceder a la perspectiva del sujeto estudiado, en comprender sus categorías, sus interpretaciones, sus percepciones y sus sentimientos. De ahí que haya sido elegida para esta investigación como estrategia fundamental de recogida de información.

Entre las diversas modalidades de entrevista, atendiendo a la estructura y al diseño, hemos elegido para el presente estudio las entrevistas semiestructuradas. Ahora bien, las preguntas que se realizan son abiertas, y permite a las personas entrevistadas la introducción de matices en sus respuestas que doten a las mismas de un valor modulador en relación a la información transmitida en primera instancia. El proceso se llevó acabo, en un primer momento se solicitó verbalmente el consentimiento a los participantes a través de la firma de un documento de consentimiento informado, en el que se explicitaba que el tratamiento de los datos no implicaría reconocer características personales, destacando el carácter totalmente anónimo de la investigación. Por este motivo no se recogieron datos de carácter personal más allá de la edad y el género que sirvieron como factores de exclusión.

Posteriormente el profesorado que accedió se les cito en su centro escolar para la realización del as entrevistas. El carácter semiestructurado de las entrevistas repercutió en la variación de las mismas, con matices de mayor y menor duración, aproximadamente de 25 minutos.

A continuación, presentamos las preguntas realizadas en la entrevista:

- ¿Qué es para ti la diversidad sexo-genérica?

- ¿Cómo se trabaja en tu centro la diversidad sexo-genérica?

- ¿Cuáles creen que son las barreras que impiden la inclusión de la diversidad sexogenérica en los centros escolares? 
- ¿Has recibido formación sobre diversidad sexo-genérica durante tus estudios de magisterio? ¿Te ha servido en tu actuación docente?

- ¿Has recibido formación sobre diversidad sexo-genérica en tu etapa de profesor en activo? ¿Te ha servido en tu actuación docente?

- ¿Has visto ofertados cursos sobre esta temática en los centros de educación del profesorado? ¿Te han interesado?

- ¿Crees que es necesario recibir formación sobre esta temática? ¿Por qué?

- ¿Te gustaría recibir formación sobre diversidad sexo-genérica? ¿Por qué?

- ¿Quieres comentar algo sobre este tema que no se haya dicho?

\section{Resultados}

Procederemos a continuación en el presente apartado a examinar la información recogida mediante las entrevistas. El proceso de análisis de los datos cualitativos, siguiendo a Pérez Serrano (1994), es sistemático y ordenado, aunque no por ello rígido, pues obedece a un plan diseñado. Esta fase de análisis de los datos obtenidos consistirá esencialmente en darle sentido a la información recopilada, descubriendo los múltiples significados que se vislumbran a través de las entrevistas realizadas.

El acercamiento al objeto de estudio requiere en primer término establecer el marco configurado por las ideas y las opiniones que tiene el profesorado sobre la diversidad sexogenérica. Con respecto a la pregunta: ¿Qué es para ti la diversidad sexo-genérica? Al respecto es posible apuntar que el profesorado entrevistado, en general, expresan escasa familiaridad con el término, e incluso señalan abiertamente que no lo tienen muy claro y que no saben qué decir. Hay muchas expresiones, como largos silencios, resoplidos, exhalaciones, que reflejan la duda sobre la temática. El conocimiento sobre la diversidad sexo-genérica es muy limitado, sus respuestas son muy escuetas, breves, titubean mucho al responder y expresan claramente que tienen muchas confusiones sobre el concepto:

Larga pausa.... (Exhalación profunda...) ¡Qué pregunta más difícil para empezar! Nunca lo he pensado. No sé la verdad, para mí la diversidad sexual. Está ahí en él.., en que cada uno es...es diferente a los, los demás y siente diferente a los demás ¿No? (Profesora, $\mathrm{n}^{\circ} 14$ ).

Pues... (Silencio largo), no sé........ No sé.... complicado... las diferentes formas de relación entre géneros (Profesora, $n^{\circ} 28$ ).

Este tema nunca lo había pensado en el colegio, la verdad es complicado (Profesor, $\left.n^{\circ} 4\right)$.

Asimismo, el profesorado formula que les resulta un tema complejo, esto nos indica que perciben la diversidad sexo-genérica como un ámbito difícil. El hecho de no considerarla en el imaginario escolar nos indica que se obvia en el discurso educativo; esto conlleva a no valorar la diversidad sexo-genérica como un tema necesario en las agendas escolares, propiciando el silencio y la negación.

Además, encontramos que piensan que la diversidad sexo-genérica remite al derecho a la libertad de elección con las personas con las que deseas mantener relaciones sexuales, y se refieren al concepto como la igualdad de derechos en las elecciones de los diversos gustos sexuales: 
Fundamentalmente que todo el mundo tenga derecho y libertad de manera normal para amar a quien quiera (Profesor, $\mathrm{n}^{\circ} \mathrm{7}$ ).

Poder elegir la opción sexual que tú decidas y tu identidad quiero decir ser libre para compartir (silencio) la diversidad sexual es que cada uno elija ser lo que quiera libremente, los mismos derechos para todo el mundo (Profesora, $\mathrm{n}^{\circ}{ }^{12}$ ).

Consideran la diversidad sexo-genérica desde el ejercicio de libertad sexual, como camino para romper la homogeneidad que la sociedad propone hasta ampliar el abanico de elección sexual. Aluden al derecho de todas las personas a poder optar desde la propia voluntad a expresar legítimamente su orientación sexual e identidad de género, apuntan que es un derecho de todas las posibles expresiones de la sexualidad.

Por otra parte, expresan que la diversidad sexo-genérica crea rechazo, y debido a ello las personas que no cumplen los roles heteronormativos sufren faltas de respeto:

Es todas las posibles formas de relacionarse sexualmente, pero también me viene la palabra intolerancia por ser diferentes, porque sé que sufren muchas faltas de respeto (Profesora, $\mathrm{n}^{\circ} 38$ ).

Continuando con la segunda cuestión: ¿Cómo se trabaja en tu centro la diversidad sexogenérica? La gran mayoría alude que poco o casi nada y apunta a que la diversidad sexogenérica es un tema tabú en los centros escolares, delicado de plantear.

Pues... (Silencio largo) si te soy sincera poco, la verdad, pero cuando hay algún problema hablamos con los niños (Profesora, $\mathrm{n}^{\circ} 3$ ).

Ni aquí ni en otros centros en los que yo he trabajado, creo que es un tema tabú, el tema de la diversidad sexual es un tabú es algo que está oculto, visibilizado (Profesora, $\mathrm{n}^{\circ} 9$ ).

Está súper oculto, eh... nadie lo quiere hablar, es como un tema tabú, es como algo que da miedo que no se trabaja vamos, un tema delicado, pero ni a nivel de las casas ni tampoco en el colegio, ni nada (Profesor, $n^{\circ} 27$ ).

El hecho de no trabajar la diversidad sexo-genérica en los centros educativos, como hemos visto reflejado anteriormente en otras categorías de análisis, refleja que es un tema prohibido, que no es tan importante como otros que sí son prioritarios para el sistema educativo. Al no educar en la diversidad sexo-genérica trasferimos conocimientos, procedimientos y valores impregnados de heterosexismo y con ello, por tanto, propiciando una escuela que rechaza esta diversidad, que no respeta el derecho a la ciudadanía sexual y, consecuentemente, perpetúa y permite condiciones de desigualdad e injusticia social hacia el alumnado no heteronormativo.

Parte del profesorado alude que es un tema que crea controversias ya desde el núcleo familiar, comentan que muchas familias rechazan trabajar esta temática en los centros escolares:

Sé por experiencias de profesores de otros centros, que las propias familias se han quejado cuando se ha trabajado la diversidad sexual (Profesora, $\mathrm{n}^{\circ} 26$ ).

Es un tema....que... bueno, la propia familia se te echa encima si hablas de él, no quieren (Profesora, $\mathrm{n}^{\circ} 24$ ).

Es interesante señalar que una profesora y un profesor concretamente, formulan que nunca a lo largo de sus trayectorias como docentes han percibido en sus aulas alumnado no heteronormativo: 
La verdad es que con las edades que yo llevo no, no he notado yo...ningún comportamiento así... la verdad que no ¡nunca! (Profesora, $n^{\circ} 1$ ).

(Silencio largo) yo no he tenido casos en mi experiencia profesional los 13 años que llevo, no he tenido experiencias profesionales en una clase, en una clase donde yo he impartido, esa edad es muy temprano... (Profesor, $n^{\circ} 27$ ).

Apuntan que en esta etapa el alumnado no expresa claramente su orientación sexual e identidad de género. Consideran el alumnado como personas asexuadas y que la sexualidad no es una dimensión básica en el desarrollo integral de la persona que tiene diferentes formas de concreción, por ello la diversidad sexo-genérica representa un aspecto de gran importancia en la formación integral de las niñas y de los niños en edades tempranas donde se forma su identidad de género y su orientación sexual, elementos fundamentales del desarrollo del alumnado. Esta "ceguera" educativa que expresan estos docentes con respecto a la diversidad sexo-genérica, negando admitir una realidad educativa, ya sea porque les dé miedo, porque no le guste o porque simplemente no quieren reconocer, enuncia la ocultación en el ámbito escolar del alumnado no heteronormativo. Incluso resulta significativo que uno de los docentes no llega a nombrar la diversidad sexo-genérica, evitando denominar los términos en su propio discurso.

Con respecto a la tercera pregunta: ¿Cuáles creen que son las barreras que impiden la inclusión de la diversidad sexo-genérica en los centros escolares? Entre las barreras que se detectan, el profesorado alude a los prejuicios sociales de la sociedad hacia la diversidad afectivo-sexual:

Pues en un primer punto (Silencio), te destacaría la mentalidad de la sociedad en la que vivimos hoy día arrastramos con muchos prejuicios (Profesora, $\mathrm{n}^{\circ} 12$ ).

Porque todo lo referente al sexo, la sexualidad, y a toda esa historia todavía eso aún no está superado a pesar de todo lo que llevamos en esta sociedad (Profesor, $n^{\circ} 35$ ).

Apuntan que el modelo imperante de la sociedad actual tiene muchos prejuicios negativos con respecto a la diversidad sexo-genérica y comentan que vivimos en una cultura marcada por los roles sexistas. Como podemos ver reflejado en las entrevistas realizadas, el profesorado denuncia el heterosexismo social que impera en nuestros días, y que conduce inevitablemente hacia la homofobia, transfobia, lesbofobia y bifobia.

Otra de las barreras que se detecta es que la diversidad sexo-genérica no es un tema preferente en las agendas educativas:

Es verdad que los colegios son como la avanzadilla tú vas viendo cosas, y esto es un paso más. Pero reconozco...Voy a decir algo muy fuerte. Así como la diversidad cultural puede tener atractivo, las comidas exóticas... esto no tiene ese atractivo, no es prioritario (Profesora, $\mathrm{n}^{\circ} 39$ ).

La verdad que hay otros temas que importan más en la escuela, este no mucho (Profesor, $\mathrm{n}^{\circ} 2$ ).

Opinan que, en el ámbito educativo al hablar de diversidad, el discurso se centra prácticamente en la diversidad funcional, la diversidad cultural, pero no la diversidad afectivo-sexual, por lo que diversidades asociadas a cuestiones de sexos, géneros, orientaciones e identidades no normativas son excluidas e invisibilizadas del ideario educativo.

Otras personas entrevistadas señalan la falta de legislación educativa específica en diversidad sexo-genérica como otra de las múltiples barreras: 
Yo creo que esa es, vamos la esencial, la básica. Y no hay ley al respecto, o sea no hay leyes, entonces tampoco tenemos a qué atenernos, ni por dónde tirar, ni en donde buscar, no hay referentes (Profesora, $n^{\circ} 24$ ).

No hay ningún marco legal que nos respalde y luego esto cara a poder justificarle a unos padres que vamos a trabajar este tema es muy importante, pero no tenemos una justificación (Profesora, $n^{\circ} 14$ ).

Si analizamos las distintas leyes educativas españolas de las últimas décadas, se refleja la escasa implicación que existe en el sistema educativo español hacia la diversidad afectivosexual. Se han producido progresos mínimos en materia de orientación sexual e identidad de género que no responden a la realidad donde la diversidad afectivo-sexual es cada vez más explícita.

Apuntan que el hecho de no tener un marco legislativo referencial, les provoca desorientación a la hora de realizar intervenciones educativas con respecto a la diversidad afectivo-sexual.

Continuando con la cuarta pregunta: ¿Has recibido formación sobre diversidad sexogenérica durante tus estudios de magisterio? ¿Te ha servido en tu actuación docente? Todos los componentes de la comunidad educativa entrevistados aluden que no han recibido ninguna formación en esta temática en los años de formación inicial en los estudios de magisterio de educación primaria:

No, ninguna formación en este tema, nada de nada (Profesora, $n^{\circ} 7$ )

No, nunca (silencio largo) nunca escuché nada de esto en la carrera (Profesor, $n^{\circ} 12$ )

No, en ninguna asignatura se trabajó este tema cuando estudié magisterio (Profesora, $\mathrm{n}^{\circ} 13$ )

La falta de formación inicial del profesorado de educación primaria se presenta como uno de los grandes obstáculos en la inclusión de la diversidad sexo-genérica. Este aspecto es un elemento clave que se refleja a lo largo de toda la investigación. Somos conscientes de la importancia de la formación en la calidad educativa, por ello la ausencia de formación en la diversidad sexo-genérica, propicia intervenciones nefastas que inciden directamente en el rechazo y discriminación del alumnado no heteronormativo. La débil formación en este tema evidencia que no es un asunto prioritario precisamente por cómo las propias escuelas y el profesorado afrontan la diversidad sexo-genérica. La ausencia de reconocimiento coloca a esta en un plano de invisibilidad que silencia el amplio abanico de expresiones identitarias, obstaculizando la inclusión de diversidad sexo-genérica.

Con referencia a la siguiente cuestión: ¿Has recibido formación sobre diversidad sexogenérica en tu etapa de profesor en activo? ¿Te ha servido en tu actuación docente?

Es muy significativo que todo el profesorado entrevistado afirma no haber recibido formación sobre diversidad sexo-genérica en la etapa de profesor en activo.

Pues concretamente en este tema, no, nunca he realizado cursos de este tema (Profesor, $\mathrm{n}^{\circ} 4$ ).

Pues como te he comentado en la anterior pregunta, no he hecho ninguna formación sobre este tema (Profesora, $n^{\circ} 39$ ).

He hecho cursos de coeducación, pero de este tema en concreto no, ninguno (Profesora, $\mathrm{n}^{\circ}$ 6). 
Deducimos que la carencia formativa es una de las causas que propician la falta de actuación frente a estas situaciones de discriminación hacia el alumnado no heteronormativo. Los números casos de acoso LGTBfóbicos, que ocurren en los centros educativos demandan urgentemente que tanto el profesorado como los demás miembros de la comunidad escolar, sin olvidar la familia, necesitan estar sensibilizados y especialmente en alerta. Por tanto, para hacer frente a esta situación de parálisis, la formación se presenta como un camino para la inclusión de las múltiples orientaciones sexuales e identidades de género.

La carencia formativa propicia trabajar esta cuestión de forma no sistemática, generando problemas tales como no lograr llegar al fondo de las cuestiones y quedarse en la superficie, no conseguir conectar con los complejos procesos personales en el que el alumnado puede estar inmerso debido al descubrimiento de su orientación sexual e identidad de género no normativa como otorgarle a la diversidad sexo-genérica un carácter excepcional, con el cual no logra integrarse en el cuerpo generalizado de conocimientos que imparte el sistema educativo.

Continuando con la quinta pregunta: ¿Has visto ofertados cursos sobre esta temática en los centros de educación del profesorado? ¿Te han interesado?

Todo el profesorado entrevistado afirma no haber visto nunca ofertados cursos sobre esta temática en los centros de educación del profesorado.

No, y hago todos los años muchos cursos (Profesor, $n^{\circ} 40$ ).

Jamás, la verdad... he visto un curso de diversidad sexual (Profesora, $n^{\circ} 6$ ).

Nunca, he visto en el CEP ofertados cursos de este tema (Profesora, $n^{\circ} 39$ ).

No poseer una buena formación específica en diversidad sexo-genérica, junto con los estereotipos sexistas que posee el profesorado, representan dos grandes hándicaps en la inclusión de la diversidad sexo-genérica. El profesorado a través del currículum oculto, impide mediante sus valores y sus actitudes el proceso de construcción de la orientación sexual y la identidad de género del alumnado no heteronormativo, pues el alumnado aprende e interioriza durante el proceso de escolarización estos patrones que determinan su identidad de género y orientación sexual. Detectamos que, por parte de las administraciones educativas, no se contempla en su oferta formativa cursos con respecto a esta temática.

Con respecto a la siguiente cuestión: ¿Crees que es necesario recibir formación sobre esta temática? ¿Por qué?

Es muy significativo que la mayoría del profesorado entrevistado afirma sobre la importancia de recibir formación como elemento clave para la inclusión de la diversidad sexo-genérica.

Sí, ipor supuesto!, es la única manera de integrarlo en clase, como no estamos formados, no sabemos actuar (Profesora, $n^{\circ} 7$ ).

Sí, es una necesidad que tenemos, por qué no sabemos hacerlo, porque no estamos formado específicamente en este tema, es necesaria para intervenir bien (Profesor, $\left.\mathrm{n}^{\circ} 12\right)$.

Sí, claro es el único camino para avanzar en este tema, la formación (Profesor, $\mathrm{n}^{\circ}$ 25).

Solamente dos profesoras no consideran importante la formación en diversidad sexogenérico, aludiendo que no es un tema prioritario en el ámbito educativo: 
No, porque con tu experiencia puedes intervenir bien, y yo necesito antes formación en otras cosas antes más prioritarias (Profesora, $\mathrm{n}^{\circ}$ 11).

No, hay otros temas más necesarios que los profesores necesitamos formación (Profesora, $\mathrm{n}^{\circ}$ 3).

Como podemos detectar el profesorado señala que no es un tema prioritario para recibir formación, estas afirmaciones nos remiten a que no consideran la diversidad sexo-genérica una cuestión importante.

Cabe resaltar que la mayoría de las personas entrevistadas defienden y expresan la necesidad de tener formación para poder actuar de manera igualitaria frente a las sexualidades no normativas, y promover una escuela realmente inclusiva con todos sus participantes. Son conscientes de que el profesorado se constituye como ejemplo a seguir para el alumnado, al ser el profesorado la figura del saber. Se detecta interés sobre el tema, reconocen sus carencias formativas y se revela que no podrían realizar una adecuada intervención en las aulas, en las que se promoviesen la inclusión en la diversidad afectivosexual entre el alumnado, al no tener formación adecuada.

Continuando con la séptima pregunta: ¿Te gustaría recibir formación sobre diversidad sexogenérica? ¿Por qué?

La mayoría demanda la necesidad de recibir formación sobre diversidad sexo-genérica como herramienta clave en la inclusión:

Sí, me gustaría formarme bien, para poder trabajar bien la diversidad sexo-genérica, (Silencio) es una carencia que tenemos y debemos reconocer. (Profesor, $\mathrm{n}^{\circ} 7$ ).

Pues sí, como te comenté anteriormente, la formación es la clave para poder intervenir bien, y por supuesto después de haber hecho esta entrevista me doy cuenta que me hace mucha falta. (Profesora, $\mathrm{n}^{\circ} 39$ ).

Sí, además creo que nos hace falta no solo al profesorado, sino a toda la comunidad educativa, porque esto es una realidad de todos (Profesor, $\mathrm{n}^{\circ} 12$ ).

Podemos detectar el interés por recibir formación en la temática basada en conocimientos, procedimientos y actitudes que transforme la escuela hacia un espacio inclusivo con las múltiples orientaciones sexuales e identidades de género, la comunidad escolar exige un nuevo modelo formativo actualizado y global que responda a las necesidades concretas de cada centro educativo.

Comentan la importancia de conectar los procesos de formación tanto del profesorado como del equipo directivo con las demandas y carencias de estos, y la necesidad de adoptar una perspectiva integral en los procesos de desarrollo profesional de todos los miembros de la comunidad educativa, en un entorno favorable para el reciclaje y la actualización profesional, para la mejora de la calidad educativa.

Para finalizar la entrevista planteamos la siguiente cuestión: ¿Quieres comentar algo sobre este tema que no se haya dicho?

Muchas personas han querido añadir algo, pues comentan que el tema es necesario visibilizarlo:

Me gustaría añadir que es un tema que estamos muy perdidos y es problemático pues no tenemos formación y además hoy en día los padres se te echan encima y debemos estar preparados (Profesora, $\mathrm{n}^{\circ} 21$ ). 
Como última cosilla, me gustaría decir que es una realidad que hay que abordar, aunque nos encontremos muchas resistencias, que es muy delicado el tema, por parte de las familias, pero también necesitamos apoyo de la administración (Profesor, $n^{\circ}$ 40).

Vuelvo a repetirlo, la clave es la formación permanente, para poder hacer las cosas bien (Profesora, $\mathrm{n}^{\circ} 39$ ).

Detectamos que el profesorado alude a la necesidad de formación para resolver "situaciones problemáticas", valoramos que la diversidad sexo-genérica debe estar incluida en el imaginario del sistema educativo como un elemento clave en el desarrollo integral de todo el alumnado, como una oportunidad de crecimiento y aprendizaje, no como un inconveniente.

Se apuesta por una formación permanente a todos los miembros de la comunidad educativa, pues señalan que al ser un tema "delicado" necesitan herramientas para superar las carencias y necesidades que obstaculizan las intervenciones pedagógicas. Por ello la formación permanente es un recurso necesario para acercar a la escuela hacia un modelo reflexivo, indagador y creativo, capaz de responder a su realidad educativa y atender a la diversidad afectivo-sexual.

\section{Conclusiones}

Tras el análisis de las entrevistas, llegamos a continuación a las reflexiones finales del estudio de investigación. Podemos concluir que la omisión discursiva de la diversidad sexogenérica reflejada a lo largo de toda la investigación, manifiesta que este tema se sigue postergando en las agendas educativas. Se refleja el modelo heteronormativo que impera en la sociedad y por ende en nuestro sistema educativo, fundamentado en los estáticos binarismos de género que atentan contra las múltiples orientaciones sexuales e identidades de género, y el ejercicio de los derechos de la ciudadanía sexual del alumnado.

Hemos podido observar en la presente investigación cómo la escuela perpetúa la construcción de la masculinidad y la feminidad hegemónica a través de un aprendizaje explícito de normas impuestas, y trasmitidas en los centros educativos. Estas ejercen una influencia determinante en la formación de la orientación sexual e identidad de género del alumnado, sobre todo en el periodo evolutivo como es la etapa de educación Primaria.

Es muy significativo que a lo largo de toda la investigación se refleja la escasa formación como hemos podido ver reflejado, deducimos que la carencia formativa es una de las causas que propician la falta de actuación frente a estas situaciones de discriminación hacia el alumnado no heteronormativo. Los números casos de acoso LGTBfóbicos, que ocurren en los centros educativos demandan urgentemente que tanto el profesorado como los demás miembros de la comunidad escolar, sin olvidar la familia, necesitan estar sensibilizados y especialmente en alerta. Por tanto, para hacer frente a esta situación de parálisis, la formación se presenta como un camino para la inclusión de las múltiples orientaciones sexuales e identidades de género.

La carencia formativa propicia trabajar esta cuestión de forma no sistemática, generando problemas tales como no lograr llegar al fondo de las cuestiones y quedarse en la superficie, no conseguir conectar con los complejos procesos personales en el que el alumnado puede estar inmerso debido al descubrimiento de su orientación sexual e identidad de género no normativa como otorgarle a la diversidad afectivo-sexual un 
carácter excepcional, con el cual no logra integrarse en el cuerpo generalizado de conocimientos que imparte el sistema educativo.

No poseer una buena formación específica en diversidad sexo-genérica, junto con los estereotipos sexistas que posee el profesorado, representan dos grandes hándicaps en la inclusión de la diversidad sexo-genérica. Como afirman Debís, Fuentes y Sparkes (2005), el profesorado a través del currículum oculto, impide mediante sus valores y sus actitudes el proceso de construcción de la orientación sexual y la identidad de género del alumnado no heteronormativo, pues el alumnado aprende e interioriza durante el proceso de escolarización estos patrones que determinan su identidad de género y orientación sexual.

Se apuesta por una formación permanente a todos los miembros de la comunidad educativa, pues señalan que al ser un tema "delicado" necesitan herramientas para superar las carencias y necesidades que obstaculizan las intervenciones pedagógicas. Por ello la formación permanente es un recurso necesario para acercar a la escuela hacia un modelo reflexivo, indagador y creativo, capaz de responder a su realidad educativa y atender a la diversidad sexo-genérica.

La formación del profesorado para la diversidad sexo-genérica será clave para desarrollar una educación inclusiva de mayor calidad para todo el alumnado si se configura como un aspecto del sistema educativo que ayuda al cambio de la cultura profesional docente en un contexto abierto a todo el alumnado y orientado por valores inclusivos. Por todo esto, abogamos por una formación del profesorado que cuestione la visión heteronormativa de la sexualidad, que le ayude a romper el binarismo de género y la concepción estática de la orientación sexual e identidad de género.

\section{Referencias}

Arnáiz Sánchez, P. (1996). Atención a la diversidad en contextos inclusivos. Comunicación presentada en el Congreso Internacional: Reto Social para el próximo milenio: Educación para la diversidad. 25 aniversario de AEDES. Madrid.

Borrillo, D. (2001). Homofobia. Barcelona: Bellaterra.

Britzman, D. (2002). La pedagogía transgresora y sus extrañas técnicas. En R. M. Mérida, (Coord.) Sexualidades transgresoras. Una antología de estudios queer (pp.197-228). Barcelona: Icaria.

Debís, J., Fuentes, J. y Sparke, A. (2005). ¿Qué permanece oculto del curriculum oculto? Las sexualidades de género y sexualidades en la educación física. Revista Iberoamericana de Educación, 21(18), 73-90.

Elsbree, A. (2002). Disruptive Pedagogies: How Teacher Educators Disrupt Homophobia. Wisconsin: University of Wisconsin.

Gallardo, F y Escolano, V. (2009). Informe diversidad afectivo-sexual en la formación de docentes. Evaluación de contenidos. Málaga: Cedma.

Goldfarb, Eva (2006). A Lesson on Homophobia and Teasing. American Journal of Sexuality Education, 1, 55-66.

Lara López, F y Ochoa Tamayo, A. (2007). Análisis del Acceso de las y los Jóvenes a los Servicios de Salud y Educación en la Región Metropolitana. Un Enfoque a Partir de la Diversidad Sexual. México: Conapred. 
Ley Orgánica 8/2013, número 295 de 9 de diciembre del 2016, para la mejora de la calidad educativa. Boletín Oficial del Estado.

López Soler, N. (2003). Curso de educación afectivo-sexual: libro de teoría. La Coruña: Netbiblo.

Pérez Serrano, G. (1994). Investigación cualitativa: retos e interrogantes: Métodos. Madrid: La Muralla.

Plummer, K. (2001). The Square of Intimate Citizenship: Some Preliminary Proposals, Citizenship Studies, 5(3), 237-253.

Powell, Richard., \& Single, H. (1996). Focus groups. International Journal for Quality in Health Care, 8(5), 499-509.

Taylor, S y Bogdan, R. (1984). Introducción a los métodos cualitativos de investigación. Barcelona: Paidós.

Vázquez Recio, R. y Angulo Rasco, F. (2003). Introducción a los estudios de casos. Málaga: Ediciones Aljibe investigación.

Wynee, N. (2008). An analysis of student teacher preparation in relation to homophobia. (Tesis doctoral inédita). Universidad del Estado de Humboldt, California. 